\title{
ELABORAÇÃO E CARACTERIZAÇÃO DE BEBIDA FERMENTADA À BASE DE EXTRATO HIDROSSOLÚVEL DE QUINOA COM POLPA DE FRUTAS
}

\author{
MILENE OLIVEIRA PEREIRA BICUDO* \\ ÉRIKA DE CASTRO VASQUES* \\ DIANA REGAZZI ZUIM* \\ LYS MARY BILESKI CANDIDO**
}

\begin{abstract}
O objetivo deste trabalho foi desenvolver bebida fermentada à base de extrato hidrossolúvel de quinoa, determinar sua composição centesimale realizaraavaliaçãosensoriale microbiológica do produto. Para o processo fermentativo foram utilizadas cepas desidratadas de Streptococcus thermophilus e Lactobacillus delbrueckii subsp. bulgaricus. O extrato hidrossolúvel de quinoa fermentado sem a adição de polpa de fruta e sacarose foi submetido às determinações de umidade, cinzas, lipídios, proteínas e carboidratos. Realizou-se o teste de aceitação e a avaliação microbiológica dos produtos, incluindo as determinações de coliformes totais, coliformes a $45^{\circ} \mathrm{C}$ e Salmonella. O produto desenvolvido alcançou boa aceitação e atendeu aos padrões microbiológicos da legislação brasileira vigente. Concluiu-se que a bebida fermentada de extrato hidrossolúvel de quinoa constitui boa alternativa para a substituição de bebidas lácteas e à base de soja.
\end{abstract}

PALAVRAS-CHAVE: Chenopodium quinoa willd.; BEBIDA FERMENTADA; COMPOSIÇÃO CENTESIMAL; ANÁLISE SENSORIAL.

* Engenheiras de Alimentos, Mestres em Tecnologia de Alimentos, Doutorandas em Tecnologia de Alimentos, Universidade Federal do Paraná (UFPR), Curitiba, PR, Brasil (e-mail: milene.oliveira@ufpr.br; erikacvas@ yafoo.com.br; dianazuim@yahoo.com.br).

** Farmacêutica Industrial, Doutora em Engenharia de Alimentos, Professora Sênior, Programa de PósGraduação em Tecnologia de Alimentos, UFPR, Curitiba, PR (e-mail: lysmary@ufpr.br). 


\section{INTRODUÇÃo}

A utilização dos alimentos como veículos de promoção do bem-estar e da saúde e, ao mesmo tempo, como redutores dos riscos de algumas doenças, tem incentivado as pesquisas de componentes naturais e o desenvolvimento de novos ingredientes e processos, possibilitando a inovação e a criação de novos nichos de mercado. Assim, cereais com alto valor nutricional e que apresentem benefícios para a saúde humana a curto, médio e longo prazo tem sido usados como matéria-prima no desenvolvimento de novos produtos alimentícios.

A quinoa (Chenopodium quinoa Willd.), pseudocereal da família Chenopodiaceae, é nativa da região dos Andes na América do Sul. A Bolívia, o Peru e o Equador são os maiores produtores de quinoa, a qual também é produzida na Colômbia, no Chile e no Brasil (TAYLOR e PARKER, 2002). A planta apresenta resistência às pragas, tendo capacidade para se desenvolver sob condições adversas de altitude e umidade, e em solos pobres em sais minerais (locais em que outros cereais não conseguem se desenvolver) (CHAUHAN, ZILLMAN e ESKIN, 1992). Devido ao seu alto valor nutritivo e balanço adequado de aminoácidos essenciais, a quinoa foi qualificada como um dos melhores alimentos de origem vegetal (SOUZA et al., 2007).

Tratando-se de pseudocereal isento de glúten, a quinoa pode ser consumida por portadores de doença celíaca sem colocar em risco sua saúde (SOUZA et al., 2007). Muitos estudos têm abordado o desenvolvimento de produtos livres de glúten, como pães, massas e produtos de confeitaria a partir de pseudocereais, considerando que o único tratamento para pacientes celíacos consiste na ausência de glúten na dieta (ALVAREZ-JUBETE, ARENDT e GALLAGHER, 2010).

Nas gôndolas dos supermercados e feiras já são encontrados produtos à base de quinoa, como barra de cereais, cookies, shakes e macarrão, dentre outros. Além dos produtos processados, a quinoa pode ser consumida in natura, na forma de grãos, flocos ou farinha.

A procura do consumidor por produtos mais saudáveis, inovadores, seguros e de prática utilização, aliada à consolidação dos produtos no mercado, contribuíram para o crescimento da indústria de bebidas fermentadas não alcoólicas, fazendo com que essas ganhassem popularidade. O processo fermentativo promove o enriquecimento biológico de substratos alimentícios por meio da produção de vitaminas, proteínas, aminoácidos essenciais e ácidos graxos essenciais, além de resultar na destruição ou redução de toxinas (STEINKRAUS, 2002).

Em face da necessidade de bebidas alternativas às de base láctea, e considerando que a fermentação auxilia no aumento do valor nutricional dos alimentos, é importante investir em pesquisas voltadas para a aplicação desse processo na produção de bebidas não alcoólicas à base de produtos de origem vegetal.

O presente trabalho teve como objetivo desenvolver bebida fermentada à base de extrato hidrossolúvel de quinoa, determinar sua composição centesimal e realizar a avaliação microbiológica e sensorial do produto.

\section{MATERIAL E MÉTODOS}

\subsection{MATÉRIAS-PRIMAS}

Foram utilizados grãos integrais de quinoa (Chenopodium quinoa Willd.), sacarose e polpa de fruta, obtidos no comércio da cidade de Curitiba.

\subsection{PROCESSAMENTO DA BEBIDA FERMENTADA}

Para a obtenção do extrato hidrossolúvel de quinoa, os grãos foram primeiramente submetidos ao aquecimento em água fervente por cinco minutos. Em seguida, os grãos foram lavados em água à temperatura ambiente e triturados com água na proporção $(\mathrm{m} / \mathrm{v})$ de grão:água 
de 1:5 em processador industrial e filtrados em peneira de 20 mesh. Devido à baixa concentração de carboidratos no extrato hidrossolúvel de quinoa, foram adicionados $0,4 \%(\mathrm{~m} / \mathrm{m})$ de sacarose ao extrato com o objetivo de servir como substrato para as bactérias láticas. Em seguida, o extrato foi submetido ao processo de fermentação.

Para o processo fermentativo foram utilizadas cepas desidratadas de Streptococus thermophilus e Lactobacillus delbrueckii subsp. bulgaricus, previamente ativadas em leite a $35^{\circ} \mathrm{C}$ e deixadas em repouso durante 20 minutos. Foram inoculados $100 \mathrm{mg} \mathrm{L}^{-1}$ de bactérias láticas em base seca ativa em relação ao volume total do extrato. Procedeu-se a fermentação do extrato em dorna de aço inoxidável de $5 \mathrm{~L}$ até que o $\mathrm{pH}$ atingisse 4,6. No extrato fermentado foram adicionadas polpas de pêssego ou uva $(18 \% \mathrm{~m} / \mathrm{m})$ e sacarose $(15 \% \mathrm{~m} / \mathrm{m})$ (PEREIRA et al., 2009).

\subsection{ANÁLISE DA COMPOSIÇÃO CENTESIMAL}

Determinou-se a composição centesimal da amostra segundo a AOAC (2000), sendo avaliados umidade, cinzas, proteínas e lipídios. O conteúdo de carboidratos foi determinado por diferença, calculando-se a média da porcentagem de água, proteínas, lipídios e cinzas, sendo o restante considerado carboidrato. Todas as determinações foram efetuadas com o extrato hidrossolúvel de quinoa fermentado sem a adição de polpa de fruta e sacarose e em triplicata.

Calculou-se o valor calórico da amostra segundo a RDC $n^{\circ} 360$, de 23 de dezembro de 2003, da Agência Nacional de Vigilância Sanitária (BRASIL, 2003).

\subsection{TESTE SENSORIAL DE ACEITAÇÃO DA BEBIDA FERMENTADA}

Realizou-se a avaliação sensorial das bebidas com a participação de 50 julgadores não treinados, recrutados como voluntários entre estudantes e técnicos da Universidade Federal do Paraná. As amostras com polpa e adoçadas (duas no total) foram servidas à temperatura de refrigeração de forma monádica, em copos de $50 \mathrm{~mL}$, codificados com três dígitos. Para a avaliação da cor, odor, sabor, textura e impressão global do produto utilizou-se a escala hedônica estruturada com 9 pontos ( 1 = desgostei muitíssimo e 9 = gostei muitíssimo), segundo Teixeira, Meinert e Barbetta (1987). As amostras foram avaliadas em cabines individuais com luz branca.

Os dados obtidos foram tabelados e submetidos à análise de variância (ANOVA) e ao teste de Tukey para determinação da diferença significativa $(p \leq 0,05)$ entre as médias das amostras, utilizando-se o programa STATISTICA, versão 7.0 (STATSOFT Inc., 2004). Adotou-se delineamento experimental de blocos casualizados, no qual cada julgador constitui uma repetição (PIMENTEL GOMES, 2000).

\subsection{AVALIAÇÃO MICROBIOLÓGICA DA BEBIDA FERMENTADA}

As análises microbiológicas foram realizadas a fim de se avaliar a qualidade higiênicosanitária dos produtos elaborados, sendo realizadas em duplicata. De acordo com a Resolução RDC n 12 (BRASIL, 2001), as bebidas devem obedecer ao seguinte padrão: bactérias do grupo coliformes a $45^{\circ} \mathrm{C}$, máximo de $10 \mathrm{NMP} / \mathrm{mL}$ e; Salmonella, ausência em $25 \mathrm{~g}$. As análises de coliformes totais, coliformes a $45^{\circ} \mathrm{C}$ e Salmonella foram efetuadas de acordo com método da AOAC (2000).

\section{RESULTADOS E DISCUSSÃO}

\subsection{COMPOSIÇÃO CENTESIMAL}

Os valores médios referentes à composição centesimal do extrato hidrossolúvel de quinoa fermentado sem adição de polpa de fruta e sacarose estão apresentados na Tabela 1. 
TABELA 1 - COMPOSIÇÃO CENTESIMAL DO EXTRATO HIDROSSOLÚVEL FERMENTADO DE QUINOA

\begin{tabular}{ccc}
\hline $\begin{array}{c}\text { Teores }\left(\mathrm{g} 100 \mathrm{~g}^{-1}\right) \\
\text { Média } \pm \mathrm{s}^{*} ; \mathrm{n}^{* *}=3\end{array}$ & Base úmida & Base seca \\
\hline Umidade & $90,9 \pm 0,31$ & - \\
Cinzas & $0,36 \pm 0,44$ & 3,96 \\
Proteínas & $3,15 \pm 0,45$ & 34,61 \\
Lipídeos & $1,2 \pm 0,53$ & 13,19 \\
Carboidratos & $4,39 \pm 0,39$ & 48,24 \\
Valor calórico $\left(\right.$ kcal. $\left.100 \mathrm{~g}^{-1}\right)$ & 40,96 & 450,10 \\
\hline
\end{tabular}

*s = Desvio padrão; **n = número de repetições.

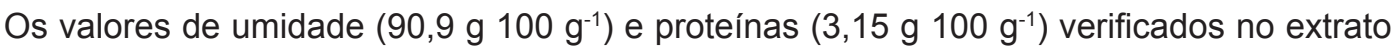
hidrossolúvel fermentado de quinoa ficaram próximos daqueles encontrados para a maioria dos

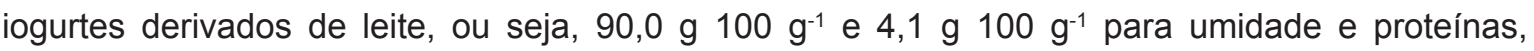
respectivamente, de acordo com a Tabela de composição de alimentos da USP (USP, 1998). Além

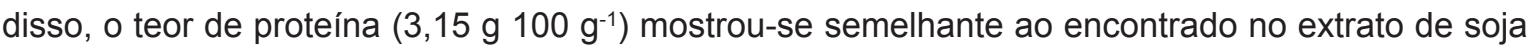
$\left(3,6 \mathrm{~g} 100 \mathrm{~g}^{-1}\right)$ estudado por Soares et al. (2010) e, comparativamente, superior ao constatado no extrato de soja (2,86 g $\left.100 \mathrm{~g}^{-1}\right)$ avaliado por Rosenthal et al. (2002). Estudo efetuado por Vilche, Gely e Santalla (2003) evidenciou concentrações de 10 a 18\% de proteína em grãos de quinoa. As diferenças encontradas em relação à composição centesimal são esperadas, devido amostras de diversas procedências, variedades, época do ano ou mesmo estádios de maturação.

De acordo com Spehar (2006), a quinoa apresenta quantidade de proteínas superior à de outros cereais como, arroz, milho e trigo. Da mesma maneira, suas proteínas são formadas principalmente por globulinas e albuminas, ricas em lisina que constitui o principal aminoácido essencial limitante na maioria dos cereais. Os teores dos aminoácidos essenciais, por serem elevados, possibilitam combinações favoráveis com cereais e leguminosas e tornam a dieta mais equilibrada. Dessa maneira, nas misturas com outros alimentos, a quinoa pode enriquecer a dieta (ASCHERI, SPEHAR e NASCIMENTO, 2002; TAYLOR e PARKER, 2002; KOZIOL, 1993). Segundo Vilche, Gely e Santalla (2003), em países como Bolívia e Peru, a quinoa é utilizada para reforçar farinhas na preparação de bolachas, macarrão e vários alimentos cozidos.

Além de representar boa fonte de proteínas, vitaminas e minerais, a quinoa constitui boa fonte de polifenois antioxidantes. Segundo Alvarez-Jubete et al. (2010), em comparação com grande variedade de frutas e vegetais, a quinoa é rica em quercetina e kaempferol, o que significa alta capacidade antioxidante e contribui para aumentar as propriedades nutritivas de alimentos que a contêm em sua composição.

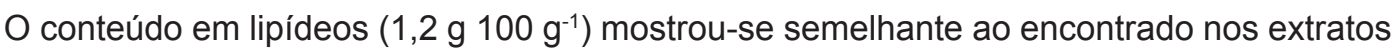
de soja estudados por Rosenthal et al. (2002) (1,5 g $\left.100 \mathrm{~g}^{-1}\right)$ e Soares et al. (2010) (1,0 g $\left.100 \mathrm{~g} \mathrm{~g}^{-1}\right)$. Segundo Koziol (1993), o teor de gordura da quinoa é similar ao da soja, mas superior ao do milho. Além disso, a quinoa é rica em ácidos graxos essenciais, principalmente os ácidos linoleico e o linolênico.

O óleo de quinoa apresenta maior estabilidade à rancidez oxidativa quando comparado ao óleo de soja, devido concentrações relativamente altas de antioxidantes naturais (tocoferois). Estudo efetuado por De Bruin (1964) com óleo refinado de quinoa mostrou concentrações de 450 ppm e 230 ppm de a-tocoferol e $\beta$-tocoferol, respectivamente. Para o óleo refinado de soja, 
Carvalho et al. (2008) encontraram valores de 180,08 ppm e 19,10 ppm de $\alpha$-tocoferol e $\beta$-tocoferol, respectivamente.

A bebida fermentada de extrato hidrossolúvel de quinoa apresentou teor de cinzas

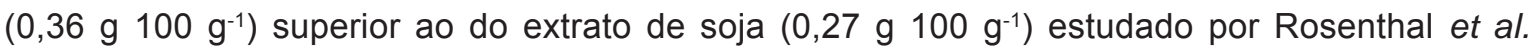
(2002). A quinoa constitui importante fonte de ferro, tendo o dobro da quantidade encontrada em cereais como cevada, trigo e arroz. A eficiência do ferro, quando administrado por meio da ingestão de quinoa alcança $74 \%$, sendo mais elevada do que a suprida pelo sulfato ferroso $(55 \%)$ (KOZIOL, 1993).

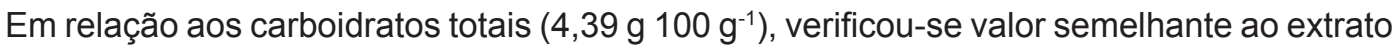

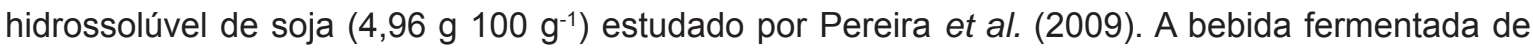
quinoa apresentou valor calórico $\left(40,96 \mathrm{kcal} \mathrm{g}^{-1}\right)$ menor que os encontrados normalmente para iogurtes convencionais como, por exemplo, o iogurte natural $(51 \mathrm{kcal})$ e a bebida láctea de pêssego (55 kcal) (NEPA, 2006). O valor calórico obtido também foi inferior ao extrato de soja $\left(71,77 \mathrm{kcal} \mathrm{g}^{-1}\right)$, extrato de arroz integral $\left(80,55 \mathrm{kcal} \mathrm{g}^{-1}\right)$ e extrato de quirera de arroz $\left(87,71 \mathrm{kcal} \mathrm{g}^{-1}\right)$ estudados por Soares et al. (2010).

Não foram encontrados na literatura relatos do aproveitamento de quinoa na elaboração de bebidas fermentadas para consumo humano. Entretanto, há citação do uso da farinha integral de quinoa associada à farinha de arroz polido para elaboração de massa alimentícia (BORGES et al., 2003). O mesmo estudo demonstrou que a farinha integral de quinoa apresenta valores superiores de proteína e fibra bruta, quando comparada à farinha de arroz polido.

\subsection{ANÁLISE MICROBIOLÓGICA}

A Tabela 2 mostra os resultados obtidos nas análises microbiológicas da bebida fermentada de quinoa, comparativamente com os valores exigidos pela RDC no 12 (BRASIL, 2001).

\section{TABELA 2 - RESULTADOS MICROBIOLÓGICOS DA BEBIDA FERMENTADA DE QUINOA $\left(n^{\star *}=3\right)$}

\begin{tabular}{ccc}
\hline Parâmetros & Dados obtidos & Legislação $^{1}$ \\
\hline Coliformes a $45^{\circ} \mathrm{C}(\mathrm{NMP} / \mathrm{mL})^{2}$ & Ausente & Máximo 10 \\
Salmonella $(\mathrm{em} 25 \mathrm{~g})$ & Ausente & Ausência \\
Coliformes totais $(\mathrm{NMP} / \mathrm{mL})$ & Ausente & - \\
\hline
\end{tabular}

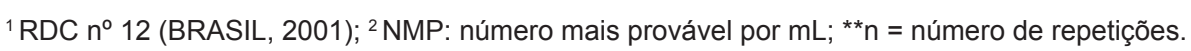

O teste realizado para contagem de coliformes a $45^{\circ} \mathrm{C}$ revelou-se negativo, não sendo necessárias as etapas seguintes de colorimetria (coliformes totais e termotolerantes). Devido ao baixo $\mathrm{pH}$ do produto, sabe-se que esses microrganismos podem sofrer estresse e não serem detectados nas análises (FORSYTHE, 2002). Contudo, a ausência de coliformes no produto final também pode indicar boas condições higiênico-sanitárias durante o processo de elaboração da bebida.

Não foi detectada a presença de Salmonella na amostra analisada, o que tornaria a bebida imprópria para o consumo. A análise para contagem de coliformes totais apresentou resultado enquadrado no padrão da RDC no 12 (BRASIL, 2001) para bebidas não alcoólicas, estabelecido como sua ausência no produto. 
Os resultados da análise sensorial das bebidas fermentadas de quinoa com polpa de fruta e sacarose estão apresentados na Tabela 3.

TABELA 3 - MÉDIAS DOS ATRIBUTOS SENSORIAIS DAS BEBIDAS FERMENTADAS DE QUINOA

\begin{tabular}{ccc}
\hline Atributos & \multicolumn{2}{c}{ Médias } \\
\cline { 2 - 3 } & Pêssego & Uva \\
\hline Aroma & $6,46^{\mathrm{a}} \pm 1,34$ & $4,72^{\mathrm{b}} \pm 1,46$ \\
Cor & $6,88^{\mathrm{a}} \pm 1,36$ & $5,74^{\mathrm{b}} \pm 1,47$ \\
Sabor & $6,64^{\mathrm{a}} \pm 1,78$ & $5,00^{\mathrm{b}} \pm 2,04$ \\
Textura & $6,34^{\mathrm{a}} \pm 1,78$ & $5,86^{\mathrm{a}} \pm 1,96$ \\
Impressão global & $6,38^{\mathrm{a}} \pm 1,71$ & $5,06^{\mathrm{b}} \pm 1,71$ \\
\hline
\end{tabular}

${ }^{a-b}$ médias com letras diferentes na mesma linha indicam diferença significativa $(p \leq 0,05)$.

A bebida fermentada de quinoa apresentou coloração clara, sabor levemente doce e viscosidade característica de iogurte. Os resultados da ANOVA da análise sensorial revelaram diferença significativa $(p \leq 0,05)$ entre as amostras em relação aos atributos aroma, cor, sabor e impressão global. Somente para o parâmetro textura não houve tal diferença. A bebida sabor pêssego apresentou boa aceitação, com média de 6,54 correspondendo ao índice de aceitabilidade superior a 70\%. Segundo Dutcoski (2007), o produto é bem aceito quando alcança índice mínimo de aceitabilidade de $70 \%$. Com relação ao número de julgadores, $85 \%$ atribuíram notas superiores a 5 em todos os atributos e $15 \%$ notas inferiores a 5 .

\section{CONCLUSÃO}

A bebida fermentada de extrato hidrossolúvel de quinoa apresentou conteúdo de proteínas semelhante ao encontrado em iogurtes lácteos. Os resultados das análises microbiológicas estão de acordo com os padrões exigidos pela legislação brasileira vigente. A avaliação sensorial mostrou que o produto elaborado teve boa aceitação, sendo a bebida com polpa de pêssego a que mais agradou aos julgadores sensoriais. A bebida fermentada de extrato hidrossolúvel de quinoa constitui opção alternativa, de alto valor nutricional, às bebidas lácteas e à base de soja.

\section{ABSTRACT}

\section{ELABORATION AND CHARACTERIZATION OF FERMENTED DRINK FROM QUINOA WATER SOLUBLE EXTRACT AND PULP FRUIT}

The aim of this work was to develop a fermented drink from quinoa water soluble extract, to determine its centesimal composition and to perform its sensorial and microbiological evaluation. Dehydrated Streptococcus thermophilus and Lactobacillus delbrueckii subsp. bulgaricus strains were applied in the fermentation process. Moisture, ashes, lipids, proteins and carbohydrates were determined. The sensory acceptance test was performed and the microbiological evaluation was done by total coliform, coliform at $45^{\circ} \mathrm{C}$ and Salmonella 
analysis. The developed product had good acceptance and achieved the microbiological standards of the legislation. The fermented drink from quinoa water soluble extract is a good alternative to replace the milk-based and soy-based drinks.

KEY-WORDS: Chenopodium quinoa Willd.; FERMENTED DRINK; CENTESIMAL COMPOSITION; SENSORY ANALYSIS.

\section{REFERÊNCIAS}

1 ALVAREZ-JUBETE, L.; ARENDT, E.K.; GALLAGHER, E. Nutritive value of pseudocereals and their increasing use as functional gluten-free ingredients. Trends in Food Science and Technology, Norwich, v. 21, p. 106-113, 2010.

2 ALVAREZ-JUBETE, L; WIJNGAARD, H.; ARENDT, E. K.; GALLAGHER, E. Polyphenol composition and in vitro antioxidant activity of amaranth, quinoa buckwheat and wheat as affected by sprouting and baking. Food Chemistry, England, v.119, p.770-778, 2010.

3 AOAC. Association of Official Analytical Chemists. Methods of analysis of the AOAC International. $17^{\text {th }}$ ed. Washington, 2000.

4 ASCHERI, J.L.; SPEHAR, C.R.; NASCIMENTO, N.E. Caracterización química comparativa de harinas instantaneas por extrusión de quinoa (Chenopodium quinoa Willd.), maíz y arroz. Alimentaria, Madrid, v.39, n.331, p.82-89, 2002.

5 BORGES, J. T. S.; ASCHERI, J. L. R.; ASCHERI, D. R.; NASCIMENTO, R. E.; FREITAS, A. S. Propriedades de cozimento e caracterização físico-química de macarrão pré-cozido à base de farinha integral de quinoa (Chenopodium quinoa, Willd) e de farinha de arroz (Oryza sativa, $L$ ) polido por extrusão termoplástica. Boletim do CEPPA, Curitiba, v. 21, n. 2, p. 303-322, 2003.

6 BRASIL. Ministério da Saúde. Agência Nacional de Vigilância Sanitária. RDC n. 12, de 02 de janeiro de 2001. Regulamento técnico sobre padrões microbiológicos para alimentos. Diário Oficial [da] República Federativa do Brasil, Brasília, DF, 10 jan. 2001, Seção I, p. 45-53.

7 BRASIL. Ministério da Saúde. Agência Nacional de Vigilância Sanitária. Resolução 360 de 23 de dezembro de 2003. Regulamento técnico sobre rotulagem Nutricional de alimentos embalados. Diário Oficial [da] República Federativa do Brasil, Brasília, DF, 26 de dezembro de 2003, Seção I, p. 33-44.

8 CARVALHO, S. M.; OGLIARI, P. J.; BARRERA-ARELLANO, D.; BLOCK, J. M. Efeito da adição de tocoferóis naturais sobre a qualidade de óleo de soja refinado e embalado em PET durante a estocagem. Braz. J. Food Technol., São Paulo, v. 11, n. 2, p. 134-143, 2008.

9 CHAUHAN, G.S.; ZILLMAN, R.R.; ESKIN, M.N.A. Dough mixing and bread making properties of quinoa wheat flour blends. International Journal of Food Science and Technology, Mysore, v. 27, p. 701-705, 1992.

10 DE BRUIN, A. Investigation of the food value of quinoa and cañihua seed. Journal of Food Science, Davis, v.29, p. 872$876,1964$.

11 DUTCOSKI, S.D. Análise sensorial de alimentos. Curitiba: Champagnat, 2007. $123 p$

12 FORSYTHE, S.J. Microbiologia da segurança alimentar. Porto Alegre: Artmed, 2002.

13 KOZIOL, M. J. Quinoa: a potential new oil crop. In: JANICK, J.; SIMON, J. E. (eds.). New crops. New York: Wiley, 1993. p. 328-336.

14 NEPA. Núcleo de Estudos e Pesquisas em Alimentação. Tabela brasileira de composição de alimentos - TACo. Versão II. 2.ed. Campinas: UNICAMP, 2006. 113 p. Online. Disponível em: <www.unicamp.br/nepa/taco/tabela. php?ativo=tabela>. Acesso em: 23 jan. 2010.

15 PEREIRA, M. O.; BAMPI, M.; RODRIGUES, F. T; DALLA SANTA, O. R.; DALLA SANTA, H. S.; RIGO, M. Elaboração de uma bebida probiótica fermentada a partir de extrato hidrossolúvel de soja com sabor de frutas. Ambiência, Guarapuava, v. 5 , n. 3 , p. $475-487,2009$.

16 PIMENTEL GOMES, F. Curso de estatística experimental. 14. ed. Piracicaba: Nobel, 2000. 477 p.

17 ROSENTHAL, A.; DELIZA, R.; CABRAL, L. M. C.; CABRAL, L. C.; FARIAS, C. A. A.; DOMINGUES, A. M. Effect of enzymatic treatment and filtration on sensory characteristics and physical stability of soymilk. Food Control, Oxford, v. 14, n. 3, p. 187-192, 2002

18 SOARES, M. J.; BASSINELLO, P. Z.; CALIARI, M.; VELASCO, P.; REIS, R. C.; CARVALHO, W. T. Bebidas saborizadas obtidas de extratos de quirera de arroz, de arroz integral e de soja. Ciência e Agrotecnologia, Lavras, v. 34, n. 2, p. 407-413, 2010. 
19 SOUZA, V.M.C.; SREBERNICH, S.; SPEHAR, C.R.; CAPRILES, V.D. Alimento perfeito. Dos Andes: pouco conhecidos, quinoa e amaranto são ricos em proteína de alto valor biológico, cálcio e outros nutrientes. Revista Metrópole do Jornal Correio Popular de Campinas, Campinas, 02 dez. 2007. p. 25-29.

20 SPEHAR, C. R. Adaptação da quinoa (Chenopodium quinoa Willd.) para incrementar a diversidade agrícola e alimentar no Brasil. Cadernos de Ciência e Tecnologia, Rio de Janeiro, v. 23, n. 1, p. 41-62, 2006.

21 STATSOFT Inc. STATISTICA for Windows. Versão 7.0. Tulsa, 2004.

22 STEINKRAUS, K. H. Fermentation in world food processing. Comprehensive Reviews in Food Science and Food Safety, San Francisco, v.1, p.23-31, 2002.

23 TAYLOR, J.R.N.; PARKER, M.L. Quinoa. In: BELTON, P.S.; TAYLOR, J.R.N. (Eds.), Pseudocereals and less common cereals: grain properties and utilization. Berlin: Springer Verlag, 2002. Chap. 3, p. 93-122.

24 TEIXEIRA, E.; MEINERT, E. M.; BARBETTA, P. A Métodos sensoriais. In: ANÁLISE sensorial de alimentos. Florianópolis: Editora da UFSC, 1987. p. 66-119.

25 Universidade de São Paulo (USP). Departamento de Alimentos e Nutrição Experimental/BRASILFOODS, Faculdade de Ciências Farmacêuticas. Tabela brasileira de composição de alimentos-USP. Versão 4.1. São Paulo, 1998. Online. Disponível em: <http://www.fcf.usp.br/tabela> Acesso em: 24 de maio 2010.

26 VILCHE, C.; GELY, M.; SANTALLA, E. Physical properties of quinoa seeds. Biosystems Engineering, v.86, n.1, p.59-63, 2003. 